\title{
Effect of Lipid Profile and Serum Creatinine on Hearing Loss in Diabetics
}

\section{Muthumagesh Arunachalam ${ }^{1}$, BP Belaldavar ${ }^{2}$ and Rajesh Radhakrishna Havaldar ${ }^{3 *}$}

${ }^{1}$ Assistant Professor, Department of ENT, Thanjavur Medical College, Thanjavur, Tamilnadu, India

${ }^{2}$ Department of ENT and Head and Neck Surgery, KAHER's J. N. Medical College, Belagavi, Karnataka, India and College of Medicine, Shaqra University, KSA ${ }^{3}$ Senior Resident, Department of ENT and Head and Neck Surgery, JN Medical College, KLE Academy of Higher Education and Research, Belagavi, Karnataka, India

*Corresponding Author: Rajesh Radhakrishna Havaldar, Senior Resident, Department of ENT and Head and Neck Surgery, JN Medical College, KLE Academy of Higher Education and Research, Belagavi, Karnataka, India.
Received: April 15, 2021

Published: : May 06, 2021

(C) All rights are reserved by Rajesh

Radhakrishna Havaldar., et al.

\begin{abstract}
Diabetes mellitus is an endocrine disorder affecting multiple organ systems of which the ear is also a vulnerable end organ. There is a proposed strong association with the sensorineural part. This study aims to find the prevalence of sensorineural hearing loss in type 2 diabetic patients and additionally assess variations of lipid profile and serum creatinine and its influence on the hearing threshold in type 2 diabetics. A cross-sectional study was conducted with a sample size of 700 at a tertiary care referral hospital over a period of 10 years. They were subjected to a detailed history and ENT examination along with Pure Tone Audiometry and relevant hematological investigations comprising of HbA1c, blood urea, and serum creatinine. Urine analysis to look for microalbuminuria was performed. Sensorineural hearing loss in type II diabetics was found to be $62.85 \%$. The total cholesterol levels were also found to be raised in hearing deprived patients. HDL and LDL levels were found to have a negligible effect on the hearing threshold. The serum creatinine levels were higher in patients with diabetes implicating an association with nephropathy. Microalbuminuria was found in nearly all patients with moderately severe to profound hearing loss. The results were statistically significant. It has been observed that short term sugar control is found to have a negligible effect over the sensorineural hearing than the long term sugar control which severely affects the neural component, as depicted by the lipid and creatinine levels in our study.
\end{abstract}

Keywords: Diabetes Mellitus; Pure Tone Audiometry; HbA1c; Sensorineural Hearing Loss; Lipid Profile; Microalbuminuria

\section{Introduction}

A new group of diseases, best termed as "disease of the plenty" has emerged as a tough challenge to the modern world's medical fraternity, of which diabetes mellitus is the foremost and notorious of all.
With a projected rise in the number of worldwide adult diabetics from 135 million in 1995 to 300 million in the year 2025, the prevalence rate would be $5.4 \%$ in 2025 [1]. In developing countries, the majority of diabetics are in the productive period of their lives, which has a major implication with respect to health care needs. The goal of modern medicine is no longer treatment of diseases 
but also their prevention and control, thereby improving the quality of life of individuals and mankind as a whole. A little above $5 \%$ of the population of the whole world (approximately 466 million) has hearing loss leading to auditory disability. Of this, about 432 million are adults. The projection is that by 2050, more than 900 million people will be victims of disabling hearing loss [2]. Hearing loss which has a marking impact on an individual, families, health system, and a country can be managed by the provision of hearing aids after a precise diagnosis. According to the World Health Organisation (WHO), only $17 \%$ of the world population which requires a hearing aid is actually using it. The rest of the $83 \%$ are not using any rehabilitation modality [2]. The pathetic situation is that one out of the forty deaf in a developing country gets a hearing aid, of which India is one [3].

The Indian population with increased susceptibility to diabetes mellitus is expected to rise up to 79.4 million double by 2030 [4]. Neuropathy is said to be prevalent in $50 \%$ of the diabetic population.

Today's otorhinolaryngologists face a massive problem in securing modern man's hearing, of which diabetes mellitus is a hindrance. The hearing loss in diabetics has a prevalence of $0-93 \%$ [5-12], characteristically a progressive, bilateral, sensorineural hearing loss predominantly in the higher frequencies by various studies [13]. There are contradictory reports such as the hearing loss being unilateral, in mid and lower frequencies as well [14].

\section{Aims and Objectives}

- To evaluate the presence of sensorineural hearing loss in type 2 diabetic patients.

- To assess variations of lipid profile and serum creatinine and its influence on hearing threshold in type 2 diabetics.

\section{Materials and Methods}

This study was a cross sectional study carried out at a tertiary care referral centre in North Karnataka region of India. The study period was from $1^{\text {st }}$ January 2009 to $31^{\text {st }}$ December 2019 i.e. 10 years.

Initially, the study was started as part of a dissertation work by the first author and later on it was continued by the remaining authors.

Sample size: Out of 748 patients who attended the ENT Out Patient Clinic, 700 with no other systemic illness except diabetes mellitus gave consent to participate in this study and undergo all the relevant investigations as planned in this study.

Sampling method: Voluntary sampling. All patients fulfiling the inclusion criteria and voluntarily agreeing to participate in the study were included in the study.

\section{Inclusion criteria}

- $\quad$ Type 2 diabetic patients on oral antidiabetic drugs.

- Patients of both the sex in age group of 30 - 55 years.

- No major systemic illness at the time of presentation.

Exclusion criteria

- Hearing loss before onset of diabetes.

- Sudden onset of hearing loss.

- History of ear discharge.

- Family history of hearing loss.

- Patients with history of head trauma, radiotherapy, ototoxic drug intake, hypertension, thyroid disorders, noise exposure.

- $\quad$ Patients with history of measles, mumps, chickenpox, rubella, meningitis, and syphilis.

Methodology

The subjects selected on basis of inclusion and exclusion criteria were subjected to routine ENT, systemic and audiometric assessment. Additionally, relevant blood tests were also performed.

Pure tone audiometry

Audiometric assessment was conducted in sound treated room delivering pure tone stimuli to one ear at a time in frequencies of $250 \mathrm{~Hz}, 500 \mathrm{~Hz}, 1000 \mathrm{~Hz}, 2000 \mathrm{~Hz}, 4000 \mathrm{~Hz}$ and $8000 \mathrm{~Hz}$ at various selected intensities as done in other studies [15]. Also, the reason for assessing in higher frequencies was due to the fact that elevated creatinine levels in chronic renal failure causes hearing loss involving the higher frequencies [16]. Masking was done when the difference in right and left unmasked air conduction threshold was $40 \mathrm{~dB}$ or more. We used MAICO MA $53^{\mathrm{TM}}$ audiometer in this study. For each audiometric frequency mean, a value greater than $25 \mathrm{~dB}$ hearing loss was considered as hearing loss [15]. 
Blood investigations

Routine blood investigations such as haemoglobin, total count, differential count and platelet count were done to rule out anemia, leukemia and other infective etiology.

Random blood sugar levels were measured using glucometer. To assess the diabetic control of the patient in the last 3 months glycosylated haemoglobin $\left(\mathrm{HbA}_{1} \mathrm{c}\right)$ was done in addition to Random blood sugar levels were measured using glucometer. HbA1c more than $6.5 \%$ or more than $48 \mathrm{mmol} / \mathrm{mol}$ was considered as diabetes [15]. The renal parameters like blood urea (Normal: 25 - $45 \mathrm{mg} /$ $\mathrm{dl}$ ) and serum creatinine (Normal: $0.7-1.5 \mathrm{mg} / \mathrm{dl}$ ) were also taken in to account. Routine urine analysis was done to monitor microalbuminuria.

The results were analysed using SPSS (Statistical Package for Social Sciences) version 23. The Pearson chi square test was used to analyse the results and $\mathrm{p}$ value was calculated to establish statistical significance.

Informed consent was obtained from each subject, after approval of the experimental protocol by the Institutional Human Ethics Committee.

\section{Results}

In our study involving 700 type 2 diabetic patients, 440 patients (62.85\%) were found to have Sensorineural Hearing Loss (SNHL) and 260 patients (37.14\%) were found normal. Among the patients suffering from SNHL only 250 patients (56.8\%) reported of hearing loss while the rest 190 patients (43.1\%) reported with other ENT complaints as seen in table 1 .

\begin{tabular}{|c|c|c|}
\hline & Subjects & Prevalence \\
\hline $\begin{array}{c}\text { Sensorineural } \\
\text { Hearing Loss } \\
\text { (SNHL) }\end{array}$ & 440 & $62.85 \%$ \\
\hline Normal & 260 & $37.14 \%$ \\
\hline
\end{tabular}

Table 1: Incidence of sensorineural hearing loss (SNHL).

The subjects involved in the study were divided into three groups as group A (31-38 years of age), group B (39-45 years of age) and group C (46-55 years of age). In group A, 38.4\% were found to be suffering from sensorineural hearing loss, in group $B$ and $\mathrm{C}$ this was found to be $64 \%$ and $71 \%$ respectively as shown in figure 1.

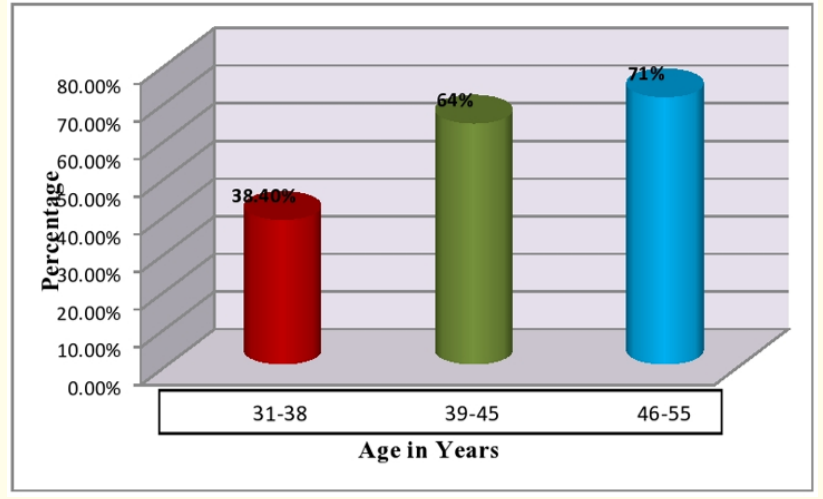

Figure 1: Graph showing percentage of SNHL (y-axis) and age group of the subject ( $\mathrm{x}$-axis).

The reason the authors used this age differentiation was that although the incidence of SNHL was $62.85 \%$, it was this younger age group where the incidence was significantly lower as compared to the other two age groups where the incidence was close to the general incidence observed. The results were statistically significant with a chi square value of 24.76 and a p value $<0.001$. In our study, there were 470 males and 230 females. The male : female ratio of SNHL was found to be 1.3:1.

The diabetic duration of the subject is taken in to account and divided into three groups of seven years duration each. It is noted that in the early years the effect of sensorineural hearing loss is less associated than the later years as suggested in figure 2 . The chi square statistic was 167.59 with $\mathrm{p}$ value $<0.0001$

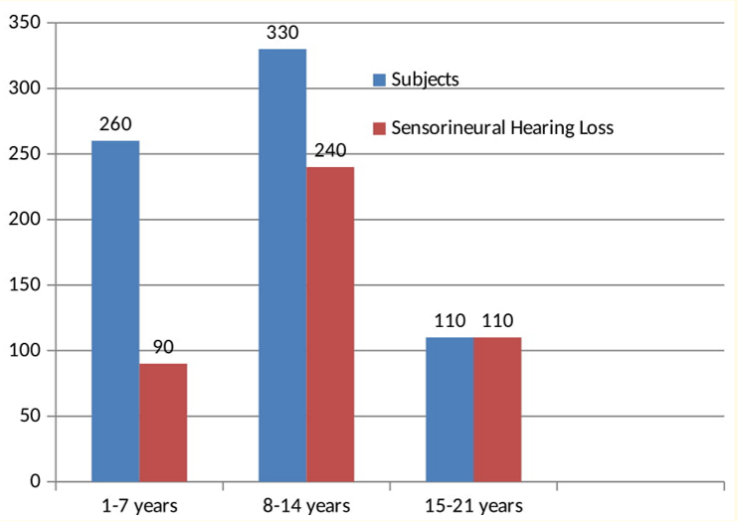

Figure 2: Graph showing number of SNHL subjects (y-axis) and duration of diabetes (x-axis). 
The random blood sugar levels were found to have no correlation between the degree of hearing loss indicating short term fluctuations of sugar levels does not hamper the hearing threshold in patients. Subjects with moderately controlled sugar levels (140 $170 \mathrm{mg} / \mathrm{dl}$ ) have increased incidence of sensorineural hearing loss (71.4\%) as compared with uncontrolled (sugar levels $>170 \mathrm{mg} /$ $\mathrm{dl}-58.6 \%$ ) and well controlled group (Sugar levels $<140 \mathrm{mg} / \mathrm{dl}$ $62.9 \%$ ). The chi-square statistics is 0.61 with a p-value of 0.4348 indicating no statistical significance.

Table 2 suggests that patients with HbA1c levels in the well controlled group is found to have more incidence of SNHL (75\%) than the uncontrolled group (64\%) and the moderately controlled group was comparatively less affected by SNHL (41.1\%). The pearson chi-square statistic is 12.79 . The $p$-value is 0.0003 . The result is statistically significant.

\begin{tabular}{|c|c|c|c|}
\hline HbA1c\% & Subjects & $\begin{array}{c}\text { Sensorineural } \\
\text { hearing loss } \\
\text { (SNHL) }\end{array}$ & Prevalence \\
\hline Less than 7 & 280 & 210 & $75 \%$ \\
\hline $7-8$ & 170 & 70 & $41.1 \%$ \\
\hline More than 8 & 250 & 160 & $64 \%$ \\
\hline $\begin{array}{c}\text { chi square- 12.79, } \\
\text { p-value:0.0003 }\end{array}$ & & & \\
\hline
\end{tabular}

Table 2: Sensorineural hearing loss (SNHL) and glycosylated haemoglobin (HbA1c).

With respect to SNHL and its relation to cholesterol levels, the uncontrolled group with more than $240 \mathrm{mg} / \mathrm{dl}$ of total cholesterol levels was more affected (87\%) with SNHL than the other two groups depicting the influence of cholesterol levels over SNHL as depicted by figure 3 . The results were statistically significant with a chi-square value of 162.99 and a p-value of $<0.001$.

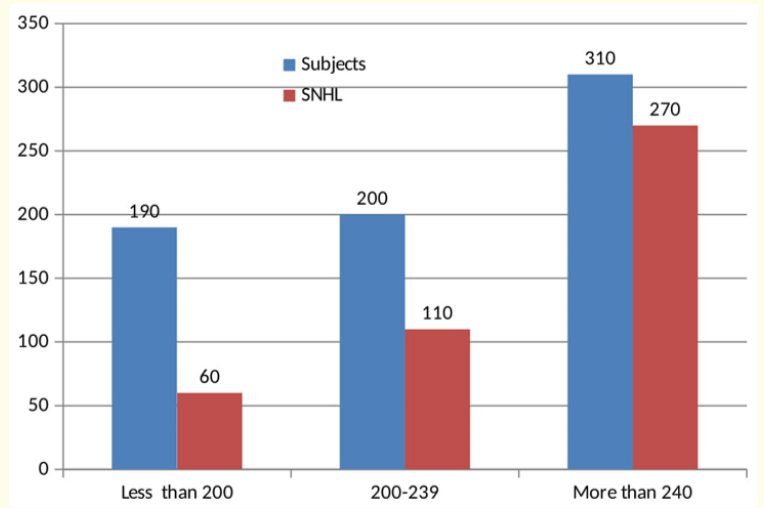

Figure 3: Graph showing number of SNHL subjects (y-axis) and cholesterol levels ( $\mathrm{x}$ - axis).
Table 3 shows that the incidence of SNHL was found to significantly increase in a proportionate manner with the triglyceride levels. This was observed in the uncontrolled group where in the serum triglycerides was found to be $>250 \mathrm{mg} / \mathrm{dl}$, the incidence of SNHL was found to be $93.9 \%$. The pearson chi-square was 61.34 and the $\mathrm{p}$ value was $<0.0001$.

\begin{tabular}{|c|c|c|c|}
\hline Triglycerides mg/dl & Subjects & SNHL & Prevalence \\
\hline $150-200$ & 110 & 30 & $27.2 \%$ \\
\hline $200-250$ & 260 & 100 & $38.4 \%$ \\
\hline More than 250 & 330 & 310 & $93.9 \%$ \\
\hline $\begin{array}{c}\text { Chi square - 61.34, p } \\
\text { value: }<0.0001\end{array}$ & & & \\
\hline
\end{tabular}

Table 3: Sensorineural hearing loss (SNHL) and triglycerides.

Table 4 suggests that the low density lipid (LDL) levels are found to have little or no direct effect on the hearing thresholds of the diabetic patients. It was observed that in the moderately controlled group (LDL range between 131 - $189 \mathrm{mg} / \mathrm{dl}$ ) sensorineural hearing loss was higher (88\%) than in the other well controlled (LDL $<130 \mathrm{mg} / \mathrm{dl}$ ) and uncontrolled groups (LDL > $190 \mathrm{mg} / \mathrm{dl}$ ). Chi square value was found to be 14.83 . The results were statistically significant among the groups with $\mathrm{p}$ value -0.0001 .

\begin{tabular}{|c|c|c|c|}
\hline LDL (mg/d) & Subjects & SNHL & Prevalence \\
\hline Less than 130 & 250 & 130 & $52 \%$ \\
\hline $131-189$ & 180 & 160 & $88 \%$ \\
\hline $\begin{array}{c}\text { More than 190 } \\
\text { Chi square - 14.83, p value: } \\
0.0001\end{array}$ & 270 & 150 & $55.5 \%$ \\
\hline
\end{tabular}

Table 4: Sensorineural hearing loss (SNHL) and low density lipoproteins (LDL).

Creatinine levels were divided in to the controlled group $(0.7$ $1.5 \mathrm{mg} / \mathrm{dl}$ ) and uncontrolled group (>1.5 mg/dl) of which, in the uncontrolled group almost all the subjects had sensorineural hearing loss as shown in table 5. The Pearson chi-square was 13.31 with a p-value of 0.0003 implying statistical significance.

\begin{tabular}{|c|c|c|c|}
\hline $\begin{array}{c}\text { Creatinine (mg/ } \\
\text { dl) }\end{array}$ & Subjects & $\begin{array}{c}\text { Sensorineural } \\
\text { hearing loss } \\
\text { (SNHL) }\end{array}$ & Prevalence \\
\hline $0.7-1.5$ & 600 & 340 & $56.6 \%$ \\
\hline More than 1.5 & 100 & 100 & $100 \%$ \\
\hline $\begin{array}{c}\text { Chi square }-13.31, \\
\text { p value: } 0.0003\end{array}$ & & & \\
\hline
\end{tabular}

Table 5: Sensorineural hearing loss (SNHL) and creatinine levels. 
In this study, 190 patients had microalbuminuria of which 180 (94.7\%) had SNHL and amongst the 510 other patients without microalbuminuria, 260(50.98\%) patients had SNHL indicating poor control of diabetes which leads to microalbuminuria, heralds sensorineural hearing loss. The pearson chi-square value was 23.36 with a p-value of $<0.0001$ confirming statistical significance.

\section{Discussion}

The diabetic influence on sensorineural hearing loss has been studied by Jorgenson (1961) with the aid of temporal bones of diabetic patients [17]. It has been reported that a unique condition is seen in diabetes mellitus wherein there is accumulation of Periodic Acid Schiff (PAS) positive substance in the modiolus vessels and capillaries of stria vascularis in various degrees [18].

The diabetic neuropathy in the auditory system was attributed to microangiopathy by Proctor in 1977, based on the hypothesis that peripheral and central neuropathies in diabetes mellitus may affect vestibular neurons [18]. Later Friedman studied diabetic patients with no complaints of decreased hearing and found a 55\% prevalence of sensorineural hearing loss involving at least one frequency [7]. Our study revealed that sensorineural hearing loss was untouched by the age of the patient and the hearing loss was similar at low and high frequencies adding to the contradictory views. Thus, the relationship between diabetes mellitus and sensorineural hearing loss is complex and under debate for many years supported by the bulk of conflicting literature. The crux about the effect of diabetes in SNHL lies centered around the cochlea and the neural pathways, which has been studied throughout the years in relation to age, sex, duration, and glycemic levels.

Out of 748 patients, after excluding patients with systemic disease, we analysed the data of 700 patients of type 2 diabetes, from various backgrounds of social life. The prevalence of sensorineural hearing loss was found to be $62.85 \%$ which is of gradual onset and progressive type. The results approximate to those that of Friedman (55\%), Aggarwal (64.86\%) and recent study done by Deepika P., et al. $(63 \%)[7,19,20]$.

The hearing loss was characteristically bilaterally symmetrical and progressive with gradual onset, however, asymmetry in the hearing loss was also noticed. The authors also observed in the audiogram of most of the patients that the hearing loss was more in the higher frequencies 4 to $8 \mathrm{kHz}$ and approximately 5 to $30 \mathrm{~dB}$ difference in the hearing threshold was observed. This result is supported by Cullen J.R and Kurien M [8,9]. Deepika P., et al. in their study have also observed that high frequency hearing loss does occur in diabetes [20]. The study results stand contrast to the results published by Tay HL in 1998, who reported hearing loss more in the mid and low frequencies [21]. However, two of the patients reported mixed hearing loss even after clearly ruling out subjects prone to conductive hearing loss in the study. As the majority of patients were under 55 years of age, the effects of presbyacusis were minimized.

On correlating sensorineural hearing loss in three age groups the incidence was more pronounced in the 46-55 years age group (71\%). In the $39-45$ years age group, it was $64 \%$ as opposed to the 31-38 years age group wherein it was 38.4\% thus, clarifying the strong association between increased age and onset of diabetes causing SNHL which shows contrast to the earlier studies carried out by Friedman and Cullen J. R [7,8]. Also, by using this age differentiation, we are able to target this particular group for earlier screening before they enter the age group wherein the debility due to deafness is going to be more pronounced. This would perhaps help us to propose screening programs at every level in the community so that these people are identified earlier and receive medical attention in time. the According to a study done in Shahroud, Iran, it was observed that the prevalence of diabetes was $16.63 \%$ and $21 \%$ in the age groups of $45-49$ years and $50-54$ years respectively [22]. This indicates that diabetes as a disease is on the rise and has started affecting young and middle age groups. Therefore, its imperative to screen these patients earlier itself for hearing loss in order to rehabilitate them at the earliest.

When compared to the younger diabetic age groups, there is a strong correlation between the duration of diabetes and SNHL in type-II diabetes. This can be observed in our study where patients with more than 8 years of duration are found to be affected as observed in the second (72.7\%) and third age groups (100\%), as compared to the younger diabetic age groups. The results were statistically significant with a $\mathrm{p}$ value $<0.001$. This is supported by the study carried out by Mehra while contradicting the other studies by Axelsson., et al. $[10,23]$. The increase in loss of hearing threshold may be attributed to the microvascular angiopathy of the inner ear, disease of the cochlear nerve, dysfunction of the outer hair cells and endolymphatic potential disruption [24]. 
The control of blood sugar levels and SNHL has been the most controversial and to ponder over this the random blood sugar levels and glycated hemoglobin $\left(\mathrm{HbA}_{1} \mathrm{C}\right)$ have been taken in to account. HbA1C was taken into consideration since it directly gives an idea about the blood sugar control of the patient in the preceding 3 months. The relation between the sugar levels and the hearing threshold in our study is inconclusive ( $p$ value 0.4348 - not siginificant) as short term sugar control cannot prove the effect on the pathology involved and may be attributed to the absence of diagnostic means to directly measure the metabolic control in the long term. In our study, the hearing loss was more in patients with well controlled HbA1c levels. Although we got these unexpected conflicting results, many studies have confirmed the same by reporting no significant relation between duration of diabetes, glycemic control and SNHL. A study done by Dosemane., et al. reported no significant correlation between HbA1c levels and hearing loss. The chi square value was 4.786 and p-value was 0.310 in their study confirming the same [24].

Lipid profile comprising total cholesterol, triglycerides, high and low-density lipids were considered in the study to probe into the pathogenesis of sensorineural hearing loss in diabetics and as an indirect measure of the metabolic control on a long term. There is a significant correlation between the increased levels of triglycerides(TGL) and SNHL, as 330 subjects with increased TGL level more than $250 \mathrm{mg} / \mathrm{dl}$ 31(93.9\%) were suffering from SNHL and those with a lower level of TGL were found to be associated with a lesser incidence of SNHL. Increased cholesterol levels are also found to raise the hearing threshold significantly in the subjects wherein $87 \%$ of them have been proven with hearing loss. The results of both the correlation of SNHL with cholesterol and SNHL with triglycerides was statistically significant with $p$ value $<0.0001$. Although we observed significant difference between the LDL groups and SNHL in our study, LDL and HDL levels were not directly contributing to the changes in the hearing threshold of the subjects involved as we observed that $88 \%$ of the patients with diabetes with moderately controlled LDL had SNHL as compared to the well controlled and poorly controlled group. This is in agreement with the meta-analysis done by Chang I.J., et al. [25]. HDL levels that are protective in nature against angiopathy were found reduced in the diabetics overall, which may contribute to the pathogenesis involved in hearing loss in diabetics. Deepika P., et al. in their study concluded that dyslipidemia had no role in causing hearing loss. The p value in their study was 0.872 for total cholesterol, 0.636 for HDL, 0.182 for LDL and 0.68 for triglycerides, when each of them was correlated with SNHL. The combination of different lipid profiles also did not have any significance. But the study was conducted on a small group of only 30 patients with DM and 30 cases of DM with dyslipidemia [20]. Swaminathan., et al. in their study found a significant hearing loss in patients with dislipidemia and diabetes. It was seen that high cholesterol, high triglycerides and higher levels of Low Density Lipoproteins (LDL) was associated with hearing loss [26].

Creatinine levels which are indicative of the long term diabetic control are found to be at high or borderline levels among the patients with SNHL. The good control group is found to have less prevalence of SNHL (56.6\%) when compared to the uncontrolled group $(100 \%)$. This result was significant with a $p$ value $<0.0001$ and is supported by the study carried out by Venkata K., et al. which strongly advocates the association of SNHL with microangiopathy. The $\mathrm{p}$ value was $<0.05$ in their study. They concluded that there is an increased incidence of SNHL with diabetes and that poor control of diabetes which is indirectly assessed by measuring increased serum creatinine values correlated positively with progressive hearing impairment [13].

\section{Conclusion}

Sensorineural hearing loss is prevalent in $62.85 \%$ of type II diabetic patients. There is a mild increase in the prevalence of SNHL as the age of the patient advances. Males are found to be more affected by the sensorineural hearing loss than their female counterparts. In the later half of the second decade of DM there is a high possibility of the patient being affected by sensorineural hearing loss. Also, short term sugar control is found to have a negligible effect over the sensorineural hearing than the long term sugar control which severely affects the neural component, as depicted by the lipid and creatinine levels.

Funding

None.

\section{Conflicts of Interest}

None. 


\section{Ethics Approval}

Approval was obtained from the ethics committee. The procedures used in this study adhere to the tenets of the Declaration of Helsinki.

\section{Consent to Participate}

Written informed consent was obtained from the relatives/ legal guardians of the study participant.

\section{Acknowledgement}

The authors would like to acknowledge Dr. Chetan Belaldavar - Department of Oral Pathology, KLES V.K.Institute of Dental Sciences, Belagavi, Karnataka, India and Dr. Umesh Nagalotimath, SAS Doctor in ENT, Countess of Chester Hospital, Chester, United Kingdom for their guidance in the final preparation of this article.

\section{Bibliography}

1. King H., et al. "Global Burden of Diabetes, 1995-2025: Prevalence, numerical estimates, and projections". Diabetes Care 21.9 (1998): 1414-1431.

2. World Health Organization. "Deafness and hearing loss".

3. World Health Organization. "Who calls on private sector to provide affordable hearing aids in developing world".

4. Kaveeshwar SA and Cornwall J. "The current state of diabetes mellitus in India". AMJ 7.1 (2014): 45-48.

5. Taylor IG and Irwin J. "Some audiological aspects of diabetes mellitus". Journal of Laryngology and Otology 92.2 (1978): 99113.

6. Abbas Naini., et al. "Effect of diabetes mellitus on the hearing ability of diabetic patients". Tanaffos 2.6 (2003): 51-58.

7. Friedman SA., et al. "Hearing and Diabetic Neuropathy". Archives of Internal Medicine 135.4 (1975): 573-576.

8. Cullen JR and Cinnamon MJ. "Hearing loss diabetics". Journal of Laryngology and Otology 107 (1993): 179-182.

9. Kurien Thomas and Bhanu TS. "Hearing threshold in patients with diabetes mellitus". Journal of Laryngology and Otology 103 (1989): 164-168.
10. Axelsson A., et al. "Hearing in diabetics". Acta Laryngology 356 (1978): 3-21.

11. Miller JT., et al. "Hearing loss in patients with diabetic retinopathy”. American Journal of Otolaryngology 4 (1983): 342-346.

12. Gibbin K P and Davis CG. "A hearing survey in diabetes mellitus". Clinical Otolaryngology 6.5 (1981): 345-350.

13. Venkata K., et al. "The effect of diabetes on sensorineural hearing loss". Otology and Neurotology 24 (2003): 382-386.

14. Shuen F W., et al. "Clinical features of sensorineural hearing loss in diabetic patients". The Laryngoscope 115 (2005): 16761680.

15. Samelli AG., et al. "Diabetes mellitus and sensorineural hearing loss: Is there an association? Baseline of the Brazilian Longitudinal Study of Adult Health (ELSA-Brasil)". Clinics (Sao Paulo) 72 (2017): 5-10.

16. Saeed HK., et al. "Sensorineural hearing loss in patients with chronic renal failure on hemodialysis in Basrah, Iraq". Tzu Chi Medical Journal 30.4 (2018): 216-220.

17. Jorgenson $\mathrm{M} B$ and Buch N H. "Studies in inner ear function and cranial nerves in diabetics". Acta Otolaryngology 55 (1961): 350-364.

18. Proctor Cornard. "Diagnosis prevention and treatment of hereditary sensorineural hearing loss". Laryngoscope 87 (1971): $35-37$.

19. Aggarwal N K., et al. "Otorhinolaryngological studies in diabetics". Indian Journal of Otology and Head and Neck Surgery 50.2 (1998): 116-120.

20. Deepika P., et al. "Does dyslipidemia worsen the hearing level in diabetics?" Journal of Otolaryngology 12.4 (2017): 198-201.

21. Tay HL., et al. "Frodo: Diabetes mellitus and hearing loss". Clinical Otolaryngology 20 (1995): 130-134.

22. Ebrahimi H., et al. "High incidence of diabetes mellitus among a middle-aged population in Iran: a longitudinal study". Can J Diabetes 40 (2016): 570-575

23. Mehra YN., et al. "Inner ear functions in diabetes mellitus with peripheral neuropathies Myers, Amsterdam New dimensions in otorhinolaryngology". Head and Neck Surgery 2 (1985): 794-799. 
24. Dosemane D., et al. "Association Between Type 2 Diabetes Mellitus and Hearing Loss Among Patients in a Coastal City of South India". Indian Journal of Otolaryngology and Head and Neck Surgery 71 (2019): 1422-1425.

25. Chang IJ., et al. "The Relationship between Serum Lipids and Sudden Sensorineural Hearing Loss: A Systematic Review and Meta-Analysis". PLoS ONE 10.4 (2015): e0121025.

26. Swaminathan A., et al. "Evaluation of the auditory effects of hyperlipidaemia and diabetes mellitus by using audiometry". Journal of Clinical and Diagnostic Research 5.8 (2011): 15281532.

Volume 5 Issue 6 June 2021

(C) All rights are reserved by Rajesh Radhakrishna

Havaldar, et al. 